\title{
Foreword to this special issue: conformal and probabilistic prediction with applications
}

\author{
Alexander Gammerman ${ }^{1}$. Vladimir $\operatorname{Vovk}^{1}$ iD
}

Published online: 8 July 2017

C) Springer International Publishing AG 2017

This issue of the Annals of Mathematics and Artificial Intelligence is devoted to conformal prediction, a modern machine learning technique that provides predictions and wellcalibrated measures of confidence for individual observations without assuming anything more than that the data are generated independently from the same probability distribution. Most of the papers in this issue have been selected from the Fifth Symposium on Conformal and Probabilistic Prediction with Applications, COPA 2016, held in April 2016 at the Centro de Investigaciones Energéticas, Medioambientales y Tecnológicas (CIEMAT), Madrid, Spain. The first Special Issue of this journal devoted to conformal prediction was published in 2015 (Volume 74, Issues 1-2); most of the papers included in that Special Issue were selected from those presented at the First Symposium in the COPA series, held in 2012 in Greece.

This new Special Issue summarises the progress of the method of conformal prediction in theory and applications. Its full title is now slightly longer, including the word "probabilistic" to reflect the importance of the method of Venn prediction (a sister technique to conformal prediction also enjoying an automatic property of being well-calibrated) and other methods of probabilistic prediction. Best papers were selected from those presented at the Fifth Symposium, and their authors were asked to revise, update, and extend their papers in order to be published in the AMAI after a new round of reviews.

The papers in this Special Issue can be divided into four groups.

- $\quad$ The invited paper (the talk being delivered by Vladimir Vapnik):

- V. Vapnik and R. Izmailov, "Knowledge transfer in SVM and neural networks”.

Vladimir Vovk

v.vovk@rhul.ac.uk

Alexander Gammerman

a.gammerman@rhul.ac.uk

1 Computer Learning Research Centre, Department of Computer Science, Royal Holloway,

University of London, Egham, Surrey, UK 
- Two papers on the theory of conformal prediction:

- V. Vovk, I. Nouretdinov, V. Fedorova, I. Petej, and A. Gammerman, "Criteria of efficiency for set-valued classification";

- V. Vovk and D. Pavlovic, "Universal probability-free prediction".

- Applications of conformal prediction are represented by six papers:

- A. Bellotti, "Reliable region predictions for automated valuation models";

- S. Zhou, E. N. Smirnov, G. Schoenmakers, and R. Peeters, "Conformal decision-tree approach to instance transfer";

- P. Toccaceli, I. Nouretdinov, and A. Gammerman, "Conformal prediction of biological activity of chemical compounds";

- H. Boström, H. Linusson, T. Löfström, and U. Johansson, "Accelerating difficulty estimation for conformal regression forests";

- $\quad$ E. Ahlberg, O. Hammar, C. Bendtsen, and L. Carlsson, "Current application of conformal prediction in drug discovery";

- C. Bendtsen, A. Degasperi, E. Ahlberg, and L. Carlsson, "Improving machine learning in early drug discovery".

- Finally, other areas of machine learning are represented by three papers:

- A. Zaytsev and E. Burnaev, "Large scale variable fidelity surrogate modeling";

- E. Burnaev, I. Panin, and B. Sudret, "Efficient design of experiments for sensitivity analysis based on polynomial chaos expansions";

- A. Kuleshov and A. Bernstein, "Nonlinear multi-output regression on unknown input manifold".

We are grateful to our sponsors, Royal Holloway, University of London, UK, and CIEMAT, Spain. Our special thanks go to Yandex (Moscow, Russia) for their help and support in organising both the Symposium and a special Alexey Chervonenkis Memorial Lecture. And finally, a big thank you to the authors of this Special Issue for their contributions, to the other participants in COPA 2016, to the members of the programme committee, and to the invited speakers, Vladimir Vapnik and Lars Carlsson. 